\title{
Shielding Charged Particle Beams
}

\author{
Klaus Dehmelt* \\ Dept. of Physics and Astronomy, Stony Brook University \\ E-mail: klaus.dehmeltestonybrook. edu
}

\begin{abstract}
Momentum measurements in the forward direction at collider experiments are inherently difficult as the deflection of charged particles to be observed requires a magnetic field component that is perpendicular to the propagation direction of those particles. This, in turn, would jeopardize the quality of the colliding beam particles. To overcome this difficulty we propose a magnetic cloak that is passively shielding the beam particles from any transverse magnetic field component and furthermore, maintain the character of the magnetic field. This would allow introducing dipole magnets in the forward region of any experiment at a collider, for instance, the Electron-Ion Collider. We present a possible setup and show the design parameters, fabrication, and limitations of a magnetic field cloak.
\end{abstract}

The 18th International Workshop on Polarized Sources, Targets, and Polarimetry, PSTP2019 23-27 September, 2019

Knoxville, Tennessee

${ }^{*}$ Speaker. 


\section{Introduction}

Most particle collider experiments make use of magnetic devices whose field component is parallel to the particle beams. This makes momentum measurements notoriously difficult when it comes to measuring the forward components of the outgoing spray of particles from the collision point. The bending of charged particles due to the magnets will only be possible due to stray field effects and this will not provide the bending power to determine the particles' momenta. One way to overcome this problem is to provide magnets in the forward region which have a field component perpendicular to the particles' path. However, this in turn would jeopardize the quality of incoming and outgoing beams. The solution to the problem is to shield charged particle beams from the surrounding magnetic field.

\section{Shielding Charged Particle Beams}

\subsection{Technology}

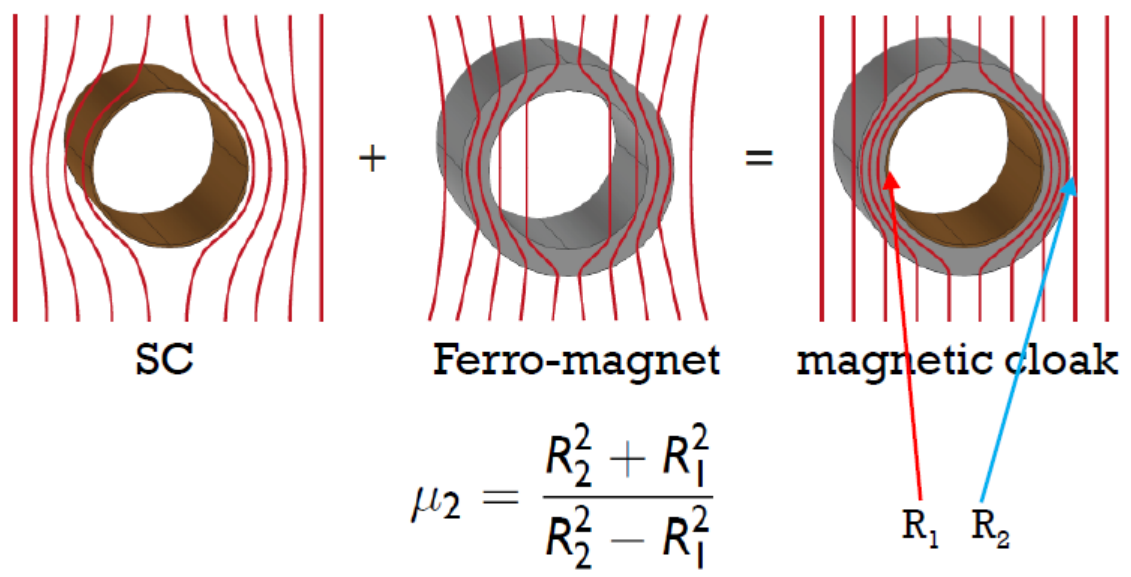

Figure 1: Merging a superconductor (SC, left) with a ferro-magnet (middle) will establish a magnetic cloak provided that proper conditions exist.

One can attempt to compensate the magnetic field lines in the region of interest, for instance with magnetic flux exclusion tubes. This technique had been applied in experiments as far as in the early 1970's at SLAC [1]. There, the aim was to suppress charged background particles that would spread out in the analyzing magnet. A superconducting flux exclusion tube of $4 \mathrm{~m}$ length and an average inside diameter of $1.3 \mathrm{~cm}$ was used to create a field free path through the magnetic analyzer. Since superconductors are pulling magnetic field lines out of their volume, such a tube would create distortions outside the tube and therefore pose a problem in momentum determination. When extorting magnetic field lines from the inside of the tube (beam pipe), one needs to attempt to compensate for the distortions outside the tube. To put both properties under one umbrella one needs to construct a combination of a superconductor and a ferro-magnet surrounding the superconductor, see Fig. 1. With the proper choice of materials one can obtain a magnetic cloaking device that is capable of creating a field-free zone in the beam pipe while maintaining the field quality surrounding it. The condition of a cloak is that the radii of the materials have to be chosen such that the 
ferro-magnet takes on a magnetic permeability [2]

$$
\mu_{r}=\frac{R_{2}^{2}+R_{1}^{2}}{R_{2}^{2}-R_{1}^{2}}
$$

Here, $R_{1}$ and $R_{2}$ are the inner and outer diameter of the ferro-magnet, respectively. Consequently, the inner diameter of the ferro-magnet corresponds to the outer diameter the superconductor.

\subsection{Experimental Realization}

We attempted the realization of above described technology with the production of a superconducting cylinder made out of rare earth barium copper oxide $\left(\mathrm{ReBCO}^{1}\right)$ and a surrounding cylinder of ferro-magnetic tape $\left(\mathrm{Fe}_{18} \mathrm{Cr} r_{9} \mathrm{Ni}\right)$. This setup was emerged in various magnetic fields as well tested for its ability to shield particle beams from magnetic fields.

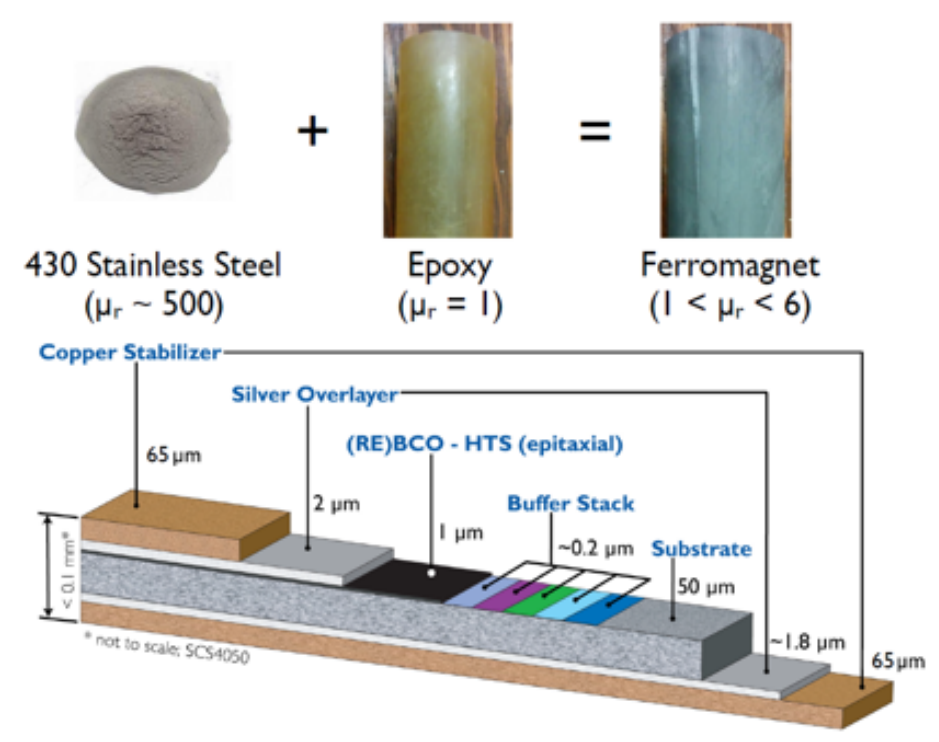

Figure 2: Top: Components of the ferromagnetic part of the magnetic cloak. Bottom: Components that make up the superconducting part of the magnetic cloak.

Ferro-Magnet The manufacturing of the ferro-magnet with customized $\mu_{r}$ depends on the fractional mass of its components. In our case, a fraction of 430-stainless-steel powder $\left(\mu_{r} \sim 500\right)$ was mixed with a fraction of commercial epoxy $\left(\mu_{r} \sim 1\right)$ [3]. The compound was mixed in a specially shaped mold and we obtained a a hardened cylinder with $1<\mu_{r}<6$ (Fig. 2, top) [4].

Superconductor The superconductor (Fig. 2, bottom) has been obtained by winding $45 \mathrm{~mm}$ wide wires made out of YBCO-ceramic ${ }^{2}$, deposited on an oxide-buffered Ni-W alloy substrate and coated with silver. The critical temperature of this material is $\sim 90 \mathrm{~K}$.

By increasing the number of layers it is possible to obtain shielding of higher magnetic fields up to a maximum when the second critical field of a superconductor is reached.

\footnotetext{
${ }^{1}$ Cuprate high temperature superconductor.

${ }^{2}$ American Superconductor Inc. www.amsc.com
} 


\subsection{Prototypes}

A set of prototypes have been constructed and tested under various conditions. The main goals for the testing were

1. Shielding ion beams from magnetic dipole fields.

2. Measure the permeability of the ferro-magnetic cylinders and test the device's cloaking ability

(a) in small magnetic fields.

(b) in moderate magnetic fields up to $0.5 \mathrm{~T}$.

For 1. the tests were performed in the Tandem van de Graaf facility at BNL and for 2a), 2b) with a Helmholtz coil and at the ANL-MRI facility, respectively.

Tandem van de Graaf Facility A $1 \mathrm{~m}$ long tube has been constructed which consisted of 2 layers of high temperature superconductive wires as described above. The length was chosen such that the tube significantly extends the magnet and not to depend on stray field effects. In Fig. 3 one can see the tube embedded in a $\mathrm{LN}_{2}$ envelope for generating the temperature needed to accomplish superconductivity. The box in the middle of the setup depicts the dipole magnet. The result from

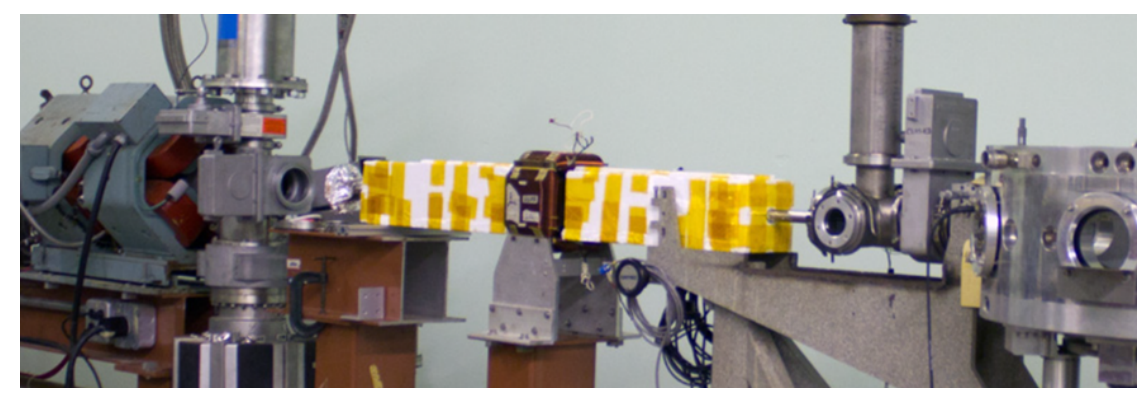

Figure 3: A shielding tube for charged particle beams, tested at the tandem van de Graaf facility at BNL.

the test can be seen in Fig. 4. The non-vanishing magnetic field as measured can be explained due to structural imperfections and trapped background fields.

Helmholtz Coil Setup In order to test the feasibility of the cloaking ability the first tests were performed with a rather weak magnet setup consisting of a pair of coils that made up a Helmholtz coil. The advantage of that setup is the high field stability and reasonable setup effort for performing a feasibility study.

For these tests a magnetic cloak was employed which consisted of a 4.5 inches long combination of a four-layer superconductor and ferro-magnet with $\mu_{r}=2.43$. The cloaking setup was arranged such that its axis was perpendicular to the coil's field direction. The results of the tests can be seen in Fig. 5. 


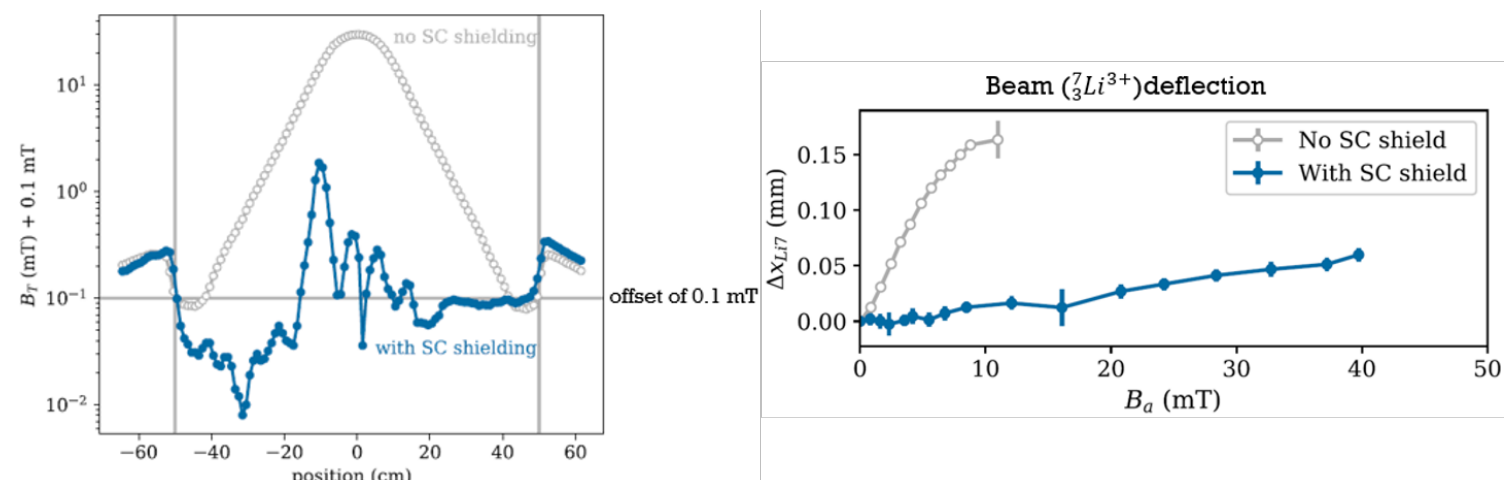

Figure 4: Shielding effect due to a superconducting cylinder around a charged particle beam pipe exposed to a dipole magnetic field. Left: remnant measured field inside the tube. Due to the logarithmic scale an offset of $0.1 \mathrm{mT}$ has been introduced. Right: deflection of a ${ }_{3}^{7} \mathrm{Li}^{3+}$ beam, with and without superconducting shield.
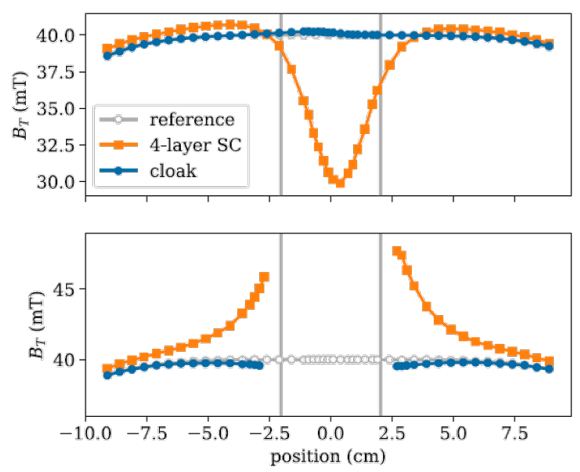
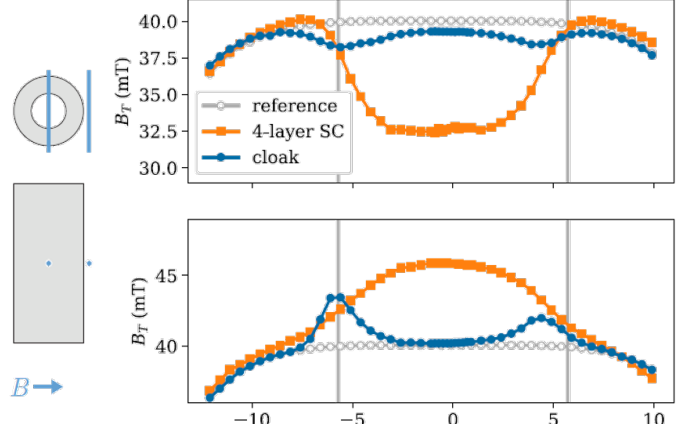

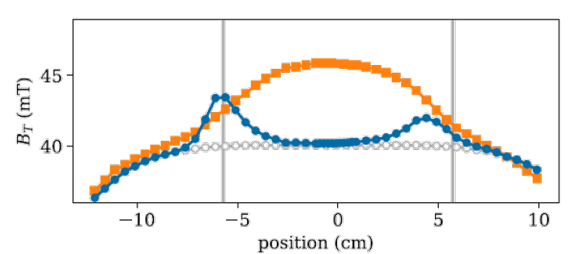

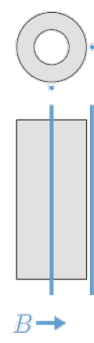

Figure 5: Cloaking within a Helmholtz-coil setup. Left: Measurement of the remnant magnetic field along the magnetic field direction along the outside (upper) and toward the center (lower) of the tube. Right: Measurement of the remnant magnetic field perpendicular to the magnetic field direction along the outside (upper) and toward the center (lower) of the tube. For comparison, a purely four-layer superconductor and the magnetic field distribution of the coil (reference) is shown.

ANL-MRI Setup The final prototype testing was performed at the 4 Tesla Magnet Facility at Argonne National Laboratory. This facility provides an MRI magnet for testing detector components and the like. The magnet has a very homogeneous field distribution and can be operated to high magnetic fields up to $4 \mathrm{~T}$. It accommodates a rail-system which allows the controlled insertion of the device under consideration and its necessary auxiliaries. A sketch of the setup can be seen in Fig. 6.

A 45 inches long cloaking device with a ferro-magnet component $\left(\mu_{r}=2.43\right)$ was placed vertically in the MRI-bore. The results of the tests can be seen in Fig. 7. Furthermore, the distribution of the magnetic field was measured away from the cloaking device (Fig. 8). 


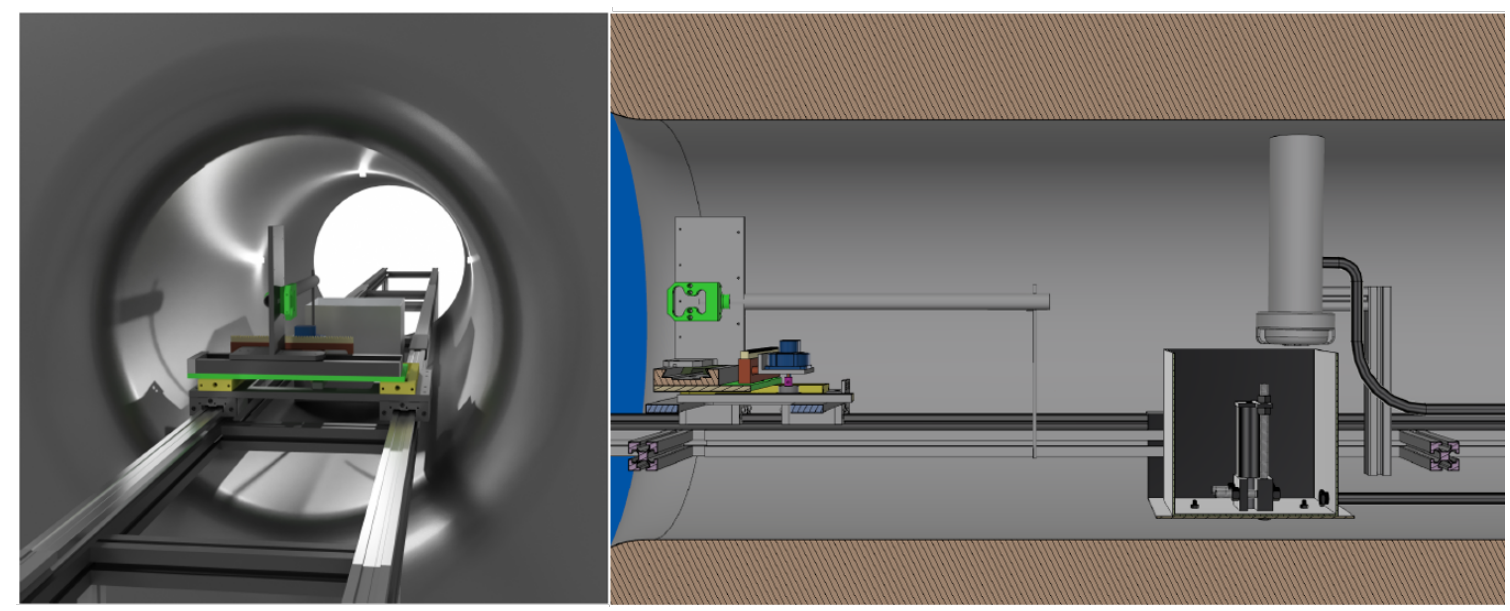

Figure 6: The rail-system for experimental access in the MRI magnet bore (left) and the cloak setup (right) which depicts the cooling box fed with $\mathrm{LN}_{2}$ and the 4.5 inch cloaking tube.
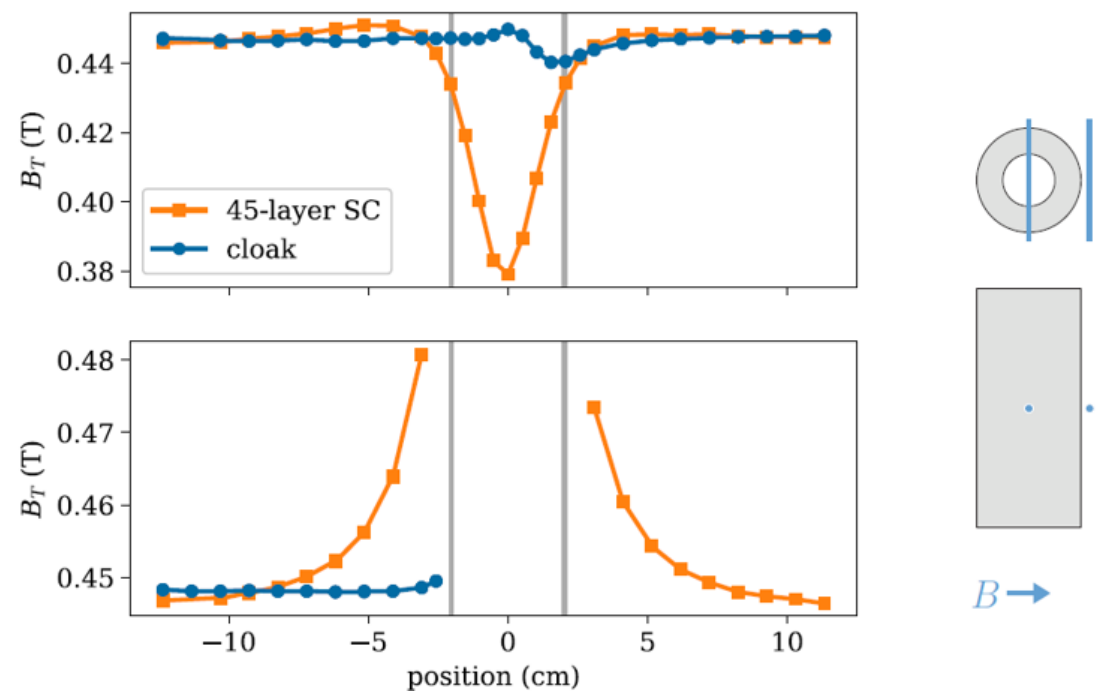

$B \rightarrow$

Figure 7: Cloaking within the ANL-MRI setup. Measurement of the remnant magnetic field along the magnetic field direction along the outside (upper) and toward the center (lower) of the tube. For comparison, a purely four-layer superconductor and the magnetic field distribution of the coil (reference) is shown. Note the scale difference (factor 10) of the magnetic field compared to Fig. 5.

\section{Conclusion}

We have demonstrated that $\mathrm{B}_{t}$ can be cloaked for magnetic fields up to $0.5 \mathrm{~T}$. The device presented here is a promising device for shielding charged particle beams but needs optimization of the manufacturing processes. One has to also carefully study for optimal parameters. The performance of the cloak can be increased when using LHe as the cooling medium as to increase the cloaking power to higher magnetic fields.

One drawback of the device is the need of a cooling device to accomplish superconducting conditions. A possible alternative might be the use of transformation optics for developing meta- 

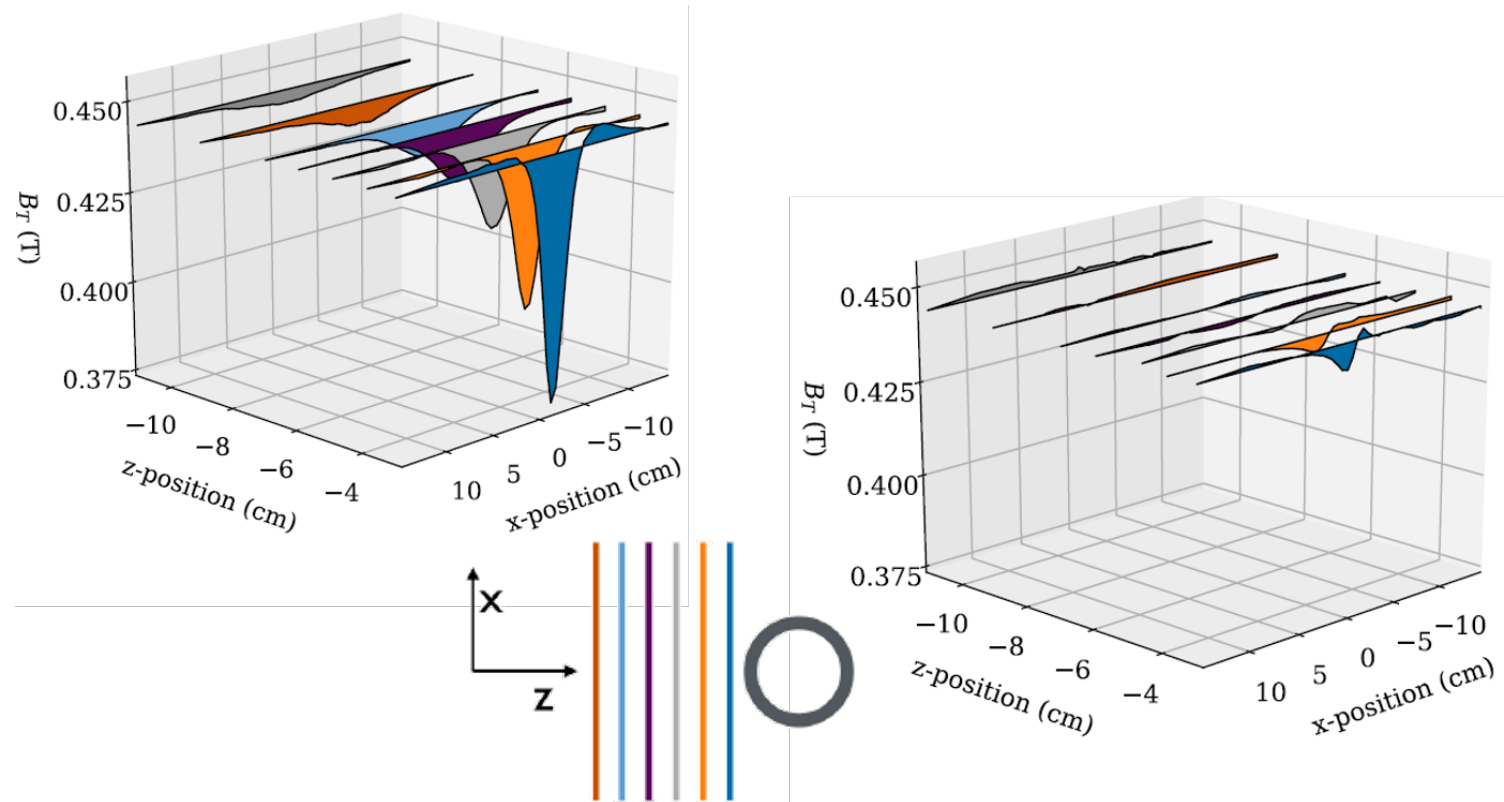

Figure 8: Far field distribution from the shielding device, for a superconducting tube only (left) and for a cloak (right).

materials and maybe use only room-temperature materials.

The work and results described in these proceedings can be seen more detailed in [5]. 


\section{References}

[1] F. Martin, S. J. St. Lorant and W. T. Toner, Nuclear Instruments and Methods 103 (1972) 503-514;

[2] F. Gomory, M. Solovyov, J. Souc, C. Navau, J. Prat-Camps, A. Sanchez, Experimental realization of a magnetic cloak, Science 335 (2012) 14661468.

[3] P. Oxley, J. Goodell, R. Molt, Magnetic properties of stainless steels at room and cryogenic temperatures, Journal of Magnetism and Magnetic Materials 321 (14) (2009) 2107 - 2114.

[4] K. N. Rozanov, A. Osipov, D. Petrov, S. Starostenko, E. Yelsukov, The effect of shape distribution of inclusions on the frequency dependence of permeability in composites, Journal of Magnetism and Magnetic Materials 321 (7) (2008) 738 - 741.

[5] K. G. Capobianco-Hogan et al., A magnetic field cloak for charged particle beams, Nucl. Instrum. Meth. A877 (2018) 149-156. 